\title{
HST Imaging of PSR1913+16
}

\author{
PHILIPPE CRANE \\ European Southern Observatory \\ Garching, Germany
}

November 14,1991

\begin{abstract}
An image of the region of the binary pulsar PSR1913+16 has been obtained with the Planetary Camera on board the Hubble Space Telescope. This image confirms previous observations of a source coincident with the radio position of the pulsar to within 0,15 arcsec. The image shows a marginal extension not seen in other so urce of similar brightness in the field.
\end{abstract}

\section{Introduction}

The binary pulsar, PSR1913+16(Hulse, and Taylor, 1975), has been claimed to be one of the best laboratories for testing general relativity(Taylor and Weisberg, 1989). This claim rests on the assumption that the objects in the binary system are both collapsed stellar remnants since only in this case will the tidal interaction between the two be negligible, and the general relativistic effects be measurable. In fact, the agreement of the observations with the predictions of general relativity are remarkably good.

Since the discovery of the binary pulsar, there have been several claims and a few disclaims of detection of an optical counterpart coincident with the radio timing position. If the claims are indeed true, then it is unlikely that the visible companion is a collapsed stellar remnant, and thus the system may not be as good a laboratory for general relativity as has been claimed. If the visible companion is part of the dynamical system of the pulsar, then the remarkable agreement of the timing data with the predictions of general relativity would be viewed as a remarkable coincidence.

The optical object was initially identified by Kristian and Westphal(1978), and an astrometric position was published by Shao and Liller(1978). A CCD image from the Kitt Peak $4 \mathrm{~m}$ was used by Crane, Nelson, and Tyson(1979) to provide further evidence of the optical coincidence. Elliot el al.(1980) also found an astrometric solution and claimed that the optical candidate does not coincide with the timing position by about 0.5 arcsec. However, they mention and discuss a systematic difference between the SAO and AGK3 star positions which is exactly what is needed to put the optical and timing position in coincidence. Subsequently, the reference star positions in the Elliot et al. paper were found to be wrong by the suspected amount(Wallace, 1991), and the optical candidate is again coincident with the radio position.

Given this background, it is very important to verify at the highest possible resolution if the object seen in the visible remains coincident with the 


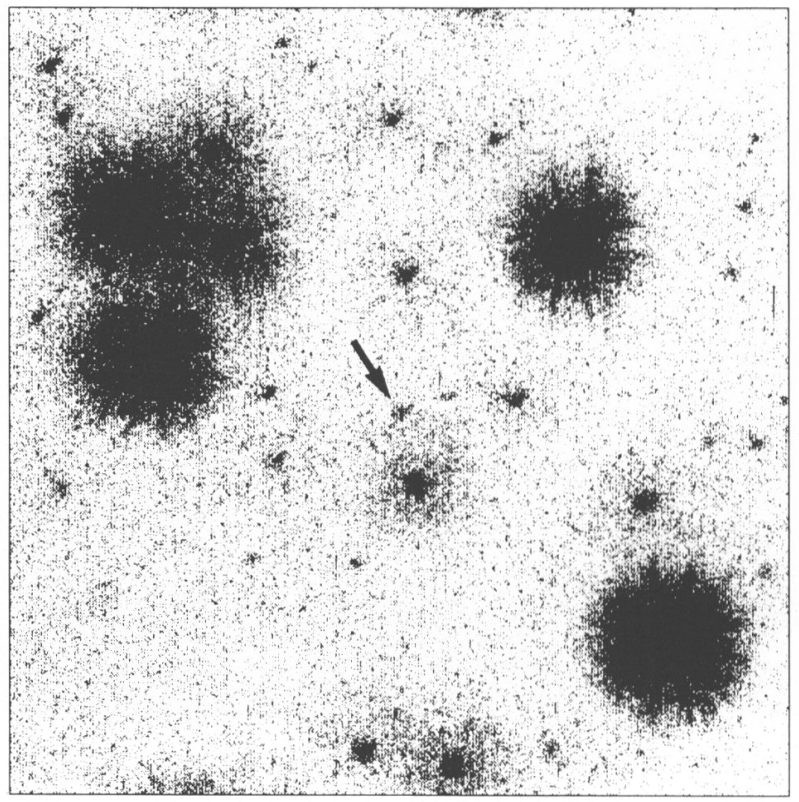

Fig. 1. Average of two 15 minute $\mathrm{PC}$ exposures through an $\mathrm{F} 606 \mathrm{~W}$ filter or the region of the binary pulsar PSR1913+16. The arrow indicates the slightly extended object which lies within 0.15 arcsec of the pulsar position.

radio timing position. Thus the HST observing program was initiated.

\section{Observations}

Two 15 minute exposures of the region of PSR $1913+16$ were obtained with the planetary camera and an F606W filter on 19 February 1991. These images were subjected to the standard data processing procedures, and summed using an alogrithm to eliminate cosmic ray hits. Several artifacts of the flat field and of the cosmic ray hits remain in the data. A careful check of a flat field frame taken through the same filter at about the same time, confirms that the flat field effects are not creating problems in the region of interest. Figure 1 shows a composite of the images where the cosmic rays have been suppressed. The position of the object suspected to be at the position of the pulsar is indicated. 
The positions of several stars in the field where determined from the centroid of the point spread function. This procedure had a typical uncertainty of about 0.3 pixels or 0.001 arcsec. A plate solution was found using the reference positions from Elliott et al.(1980) corrected according to Wallace(1991). This solution had an uncertainty of 0.06 arcsec. This yields an optical position in the FK4 B1950.0 system (Equinox 1950.0 Epoch 1978.58) of

$$
\begin{gathered}
\text { R.A. }=19^{h} 13^{m} 12 . .^{s} 440 \\
\text { Dec. }=16^{\circ} 01^{\prime} 8 . " 05
\end{gathered}
$$

This position must be compared with the radio timing position corrected for the e-terms of aberration of:

$$
\begin{gathered}
\text { R.A. }=19^{h} 13^{m} 12 .^{s} 442 \\
\text { Dec. }=16^{\circ} 01^{\prime} 8 .^{\prime \prime} 17
\end{gathered}
$$

The radio position is for Equinox 1950.0 Epoch 1984.52 and is the position from Taylor and Weisberg(1989) with e-terms removed and without accounting for proper motion. This result puts the optical and radio positions within 0.13 arcsec of one another. The errors in the optical position are formally only 0.06 arcsec, but this does not included systematic errors as discussed by Elliot it et al.(1984). Also, the errors in the radio timing position are formally quite small, but again the systematic differences between the ephemerides is about 0.15 arcsec (Backer et al., (1985). In addition there are uncertainties of at least 0.1 arcsec in the alignment of the radio and optical coordinate systems. Thus the optical and radio positions agree with each other within the errors, and the possibility that the detected object is physically associated with the pulsar must be taken seriously.

\section{References}

Backer, D.C., Fomalont, E.B., Goss, W.M., Taylor, J.H., Weisberg, J.M., 1985, A.J.,

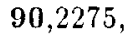

Crane, P., Nelson, J.E., and Tyson, J.A., 1978, Nature,280,367.

Elliot, K.H., Peterson, B.A., Wallace, P.T., Jones, D.H.P. Clements, E.D., Hartley, K.F., Manchester, R.N., 1980, MNRAS, 192, 3P.

Hulse, R.A., and Taylor, J.H., 1975, Ap.J.(Letters), 195,L51.

Kristian, J. and Westphal,1978, J.A., I.A.U. Circ., No.3242.

Shao, C., and Liller, W., 1978, Ap.J.,223,266.

Taylor, J.H. and Weisberg, J.M., 1989, Ap.J.,345 439.

Wallace, P.T., 1991, private communication. 